\title{
The effectiveness of manual and powered toothbrushes
}

\begin{abstract}
Proper oral hygiene is a basis for preventing dental caries and periodontal diseases. The most popular method of mechanical plaque removal is brushing teeth using manual and powered toothbrushes. The review of literature shows that removal of dental calculus using the powered toothbrushes is more effective, what is supported by greater reduction of plaque and symptoms characteristic for soft tissue inflammation. It needs to be underlined that the effectiveness of tooth brushing, regardless of the kind of toothbrush, to a great extent depends on the frequency and technique of brushing, on the time devoted to this hygienic procedure, and on the type of toothbrush bristles.
\end{abstract}

Keywords: oral hygiene, powered toothbrush, manual toothbrush.

DOI: $10.1515 /$ pjph-2017-0027

\section{INTRODUCTION}

Oral conditions, including dental caries and periodontal diseases, may have a negative impact on the general health condition of an organism. That is why, it is essential to take care of oral health [1]. Oral health condition depends on many factors, such as age, sex, genetic factors, or social status, however, a direct factor which initiates pathological processes is bacterial and fungal flora [2]. It has been proven that accumulation of dental plaque is the cause of gingivitis, and the removal of plaque leads to the relief of inflammatory conditions [3]. Professional teeth cleaning, as well as therapeutic procedures concerning teeth and periodontal diseases, help to maintain oral health; although the most important, preventive factor is everyday proper oral hygiene.

There are various procedures aiming at control of amount of bacterial plaque, and the most popular one is brushing teeth with toothpaste containing fluorine [4-8]. A lot of people additionally use dental flosses, interdental brushes, oral irrigators, tongue scrapers and mouth rinses [4-7]. It has been proven that extra hygienic procedures, such as flossing [4-6] and using mouthwashes [9-10] have significant influence on reduction of plaque.

Oral hygiene has already been important over 5000 years ago, and at that time there appeared the first devices for tooth brushing. However, it is thought that they are Chinese people, during the Tang Dynasty (ruling between the years 618 and 907), who constructed the first toothbrush composed of the bristles and a handle. In 1780, William Addis designed and created the first toothbrush called The First Modern Toothbrush [11].
In turn, the first powered toothbrush was invented in 1939 in Switzerland by Philippe-Guy Woog. Nonetheless, it only became popular in 1960s, especially in the United States. Nowadays, also in Poland, there is wide range of toothbrushes available, both manual and electric or sonic ones.

The manual toothbrushes are the ones that are most often used. While purchasing, the buyers are guided by custom, price, hardness of the brush bristles, availability, advertising spots [3], dentist's opinion, arrangement of the bristles, even though in the case of the latter, there is no significant difference when it comes to bacterial plaque elimination [12]. The electric toothbrushes can be divided into oscillating/rotating/ pulsating (modification of oscillating-rotating), sonic and ultrasonic ones. The oscillating/rotating/pulsating toothbrushes have a circular head, which moves by rotation in a clockwise direction and in the counter-clockwise direction with the frequency up to 40,000 rotations per minute. The sonic toothbrushes have an elongated head and make up to 31,000 brush-strokes per minute. The ultrasonic toothbrushes generate ultrasound pulses with the frequency equal to 96 million ultrasonic pulses per minute.

One of the first review papers concerning comparison of effectiveness of plaque removal and oral health, was the publication, which was created in cooperation with the Cochrane Oral Health Group in 2003, and was later updated in 2005, 2011, and finally in 2014. The authors searched the following databases: 'The Cochrane Oral Health Group's Trials', 'The Cochrane Central Register of Controlled Trials', 'MEDLINE', 'EMBASE' and 'CINAHL', looking for randomized studies concerning comparison of the powered toothbrushes

\footnotetext{
${ }^{1}$ Chair and Department of Public Health, Medical University of Lublin, Poland

${ }^{2}$ Department of Dental Prosthetics, Medical University of Lublin, Poland

${ }^{3}$ Chair and Department of Paedodontics, Medical University of Lublin, Poland
} 
with the manual ones, with physically fit participants who brushed teeth for, at least, four weeks. Initially, 1195 studies have been identified, out of which only 51 fulfilled the criteria of inclusion into a meta-analysis. The chosen studies involved a total of 4624 participants. The analysis did not include papers which lacked: a specified kind of toothbrush used by participants, various (authors' own) indexes of oral hygiene and gums inflammation evaluation, unclearly shown results, assessment of only a part of oral cavity (sextant/quarter of dentition). The results of the studies unequivocally show that the electric oscillating-rotating toothbrushes guarantee statistically significant advantage, compared to the manual ones. Both plaque and gingivitis were to a greater degree reduced when using oscillating-rotating toothbrush in a short-term observation (up to 3 months), as well as in a long-term one (more than 3 months) [13].

In 2010 Klukowska et al. examined 118 persons with the symptoms of gums inflammation and with present bacterial plaque. At the beginning of the research and after four weeks of its duration, the Modified Gingival Index (MGI) and the Gingival Bleeding Index (GBI) were evaluated, and the presence of plaque was examined using the Navy Plaque Index in the Rustogi modification (RMNPI). The results of the studies included into the systematic review of studies show, that in the group of respondents using oscillating/rotating/pulsating toothbrush, there was a significant improvement of oral health. When it comes to persons using electric toothbrushes, it was found that reduction of symptoms of gums inflammation was three times bigger, and that reduction of gingival bleeding index was two times more effective, compared to persons using manual toothbrushes [14].

In the later study, the above-mentioned author et al. compared effectiveness of the manual and electrical toothbrushes. The patients included into the study had to have at least 16 teeth, could not be periodontally nor orthodontically treated, could not have more than 5 active cavities and did not use removable dentures. The respondents were randomized into two groups; in one of them, participants used electric toothbrushes, and in the other one, the manual ones with soft bristles. In both groups the same toothpaste was used and all the individuals brushed teeth twice a day for 2 minutes. Reduction of bacterial plaque was found out in all the patients. However, in the case of respondents using the electric toothbrushes, the reduction of bacteria in the whole oral cavity was bigger by $7.9 \%$, compared to those who used the manual ones [15].

In a paper published in 2017, there compared effectiveness of dental plaque control and gums inflammation in patients using a manual toothbrush (Oral-B40 Regular Advantage Plus) and an electric one (Brown/Oral-B two-dimensional), on the basis of the value of plaque index (PI), oral hygiene index (OHI) and gingival index (GI). The research included 45 patients between 19 and 23 year old, who did not have any active caries nor found parafunctions, did not smoke, had not used electric toothbrushes before, had at least 20 teeth and did not take medications for systemic diseases. The respondents were supposed to clean their teeth with manual toothbrushes using the Bass method or with electric toothbrushes, for 28 days. Both groups used the same toothpaste. Every seven days, the patients had their indexes evaluated. In the case of manual toothbrushes, the reduction of PI was approximately 0.54 (from 0.71 to 0.17 ), and in the case of powered ones, it was 0.687 (from 0.70 to 0.013 ). In terms of GI reduction, better results were also achieved by persons using electric toothbrushes - from 0.29 (on the first day of the study) to 0.00 (on the last day of the study), compared to patients using manual toothbrushes who achieved reduction from 0.39 to 0.05 . The results showed that in both groups there was reduction of PI, OHI and GI values, however, in the case of the group using powered toothbrushes, the reduction was significantly bigger [16].

Sharma et al. evaluated effectiveness of a new oscillatingrotating toothbrush head, Oral-B Precision Clean, in removing dental plaque, in comparison with a manual toothbrush recommended by the American Dental Association. Initially, the respondents colored their plaque with staining solution, which made an assessment of bacterial plaque level easier. Then, they brushed their teeth with either manual or electric toothbrush for two minutes in accordance with the manufacturers' recommendations. The plaque level was evaluated immediately after brushing. It turned out that both toothbrushes contributed to the reduction of bacterial plaque, although the electric one - significantly [17].

In the three-month study, Barnes et al. assessed an influence of the manual and electric toothbrushes on the reduction of gums inflammation and bacterial plaque. The research included 70 persons who were classified after a preliminary examination concerning presence of gums inflammation, bacterial plaque and soft tissue injuries. The participants were randomized into two groups using different toothbrushes (manual and electric) and were given instructional videos presenting proper tooth brushing. Additionally, after the first visit, each participant had his/her teeth professionally cleaned. After 12 weeks of study, the patients were reexamined to evaluate their condition of gums inflammation and presence of bacterial plaque. The inflammatory condition was reduced in the group using electric toothbrushes, and remained on a similar level in the group using manual toothbrushes. In case of both toothbrushes, no soft tissue gums injuries nor bacterial plaque reduction were observed [18].

In the case of both, manual and electric toothbrushes, the crucial are the movements the patient performs during brushing teeth. Guns et al. [19] recorded 100 study participants, who informed in the survey that they use electric toothbrush at home. In the study, each of them brushed teeth with both manual and electric toothbrush, and the sequence was randomized. The camera was placed behind the one-way mirror, what allowed respondents to brush teeth with anyone else being present, as it could influence the final result. The examined tended to brush teeth longer with the electric toothbrushes (averagely 145 seconds) than with manual ones (averagely 135 seconds). Analyzing the surface of brushed teeth, the authors proved that, when using manual toothbrush, all the persons $(100 \%)$ cleaned palatal surface, while in the case of electric toothbrush, not all the respondents $(96.8 \%)$ managed to properly clean this surface. While using manual toothbrushes, the respondents most often performed circular and horizontal movements $(88 \%)$. The same kinds of movement were performed by over half of respondents while using electric toothbrush $(50.5 \%)$. Only $21.1 \%$ of patients for most of the time performed passive brushing using an electric toothbrush. As the results of the studies show, replacement of a manual toothbrush with an electric one may not bring the expected outcomes unless patients simultaneously change the movements performed during tooth brushing. 
A dentist or a dental hygienist should give advice on the method of proper tooth brushing, including, among others, kind of used by a patient toothbrush - either manual or electric one. Effectiveness of cleaning teeth and possible side effects of improper technique of tooth brushing need to be controlled, and where necessary, corrected during following dental appointments.

\section{CONCLUSIONS}

Summing up, it needs to be concluded that tooth brushing is the most popular and commonly accepted way of taking care of proper oral hygiene. Tooth brushing, regardless of a type of toothbrush - manual or electric one, is an effective way of controlling bacterial plaque, which is responsible for dental caries and periodontal diseases, especially gums inflammation. The electric toothbrushes better remove dental calculus, compared to the manual ones. Nevertheless, the effectiveness of tooth brushing, regardless of the kind of toothbrush, to a great extent depends on the frequency and technique of brushing, on the time devoted to this hygienic procedure, and on the type of toothbrush bristles.

\section{REFERENCES}

1. Ash MM, Githin BN, Smith WA. Correlation between plaque and gingivitis. J Periodontol. 1964;35:424-9.

2. Fejerskov O, Manji F. Risk assessment in dental caries. In: JD Bader (ed). Risk assessment in dentistry. Chapel Hill, NC: University of North Carolina Dental Ecology, 1990;215-7.

3. Loe H, Theilade E. Jensen SB. Experimental gingivitis in man. J Periodontol. 1965;36:177-87.

4. Wilder RS, Bray KS. Improving periodontal outcomes: merging clinical and behavioral science. Periodontology. 2000;71(1):65-81.

5. Claydon NC. Current concepts in toothbrushing and interdental cleaning. Periodontology. 2000:48(2008);10-22.

6. Sgan-Cohen HD. Oral hygiene: past history and future recommendations. Int J Dent Hyg. 2005;3(2):54-8.

7. Westfelt E. Rationale of mechanical plaque control. J Clin Periodontol. 1996;23(3 Pt 2):263267.

8. Twetman S, Axelsson S, Dahlgren H, et al. Caries-preventive effect of fluoride toothpaste: a systematic review. Acta Odontol Scand. 2003;61(6):34755.

9. Marinho VCC, Higgins JPT, Logan S, Sheiham A. Fluoride mouthrinses for preventing dental caries in children and adolescents. Cochrane Database Syst Rev. 3. 2003;1. [http://dx.doi.org/10.1002/14651858.CD002284].

10. Marinho VCC, Higgins JPT, Sheiham A, Logan S. Combinations of topical fluoride (toothpastes, mouthrinses, gels, varnishes) versus single topical fluoride for preventing dental caries in children and adolescents. Cochrane Database of Systematic Rev. 2004;1: CD002781. DOI: 10.1002/14651858. CD002781.pub2

11. Kumar JV. Oral hygiene Aids. In: S. S. Hiremath (ed). Textbook of preventive and community dentistry. 2nd ed. Elsevier. 2011:412-27.

12. Sripriya N, Shaik Hyder Ali KH. A comparative study of the efficacy of four different bristle designs of tooth brushes in plaque removal. J Indian Soc Pedod Prev Dent. 2007;25:76-81.

13. Yaacob M, Worthington HV, Deacon SA, et al. Powered versus manual toothbrushing for oral health. Cochrane Database of Systematic Rev. 2014;6: CD002281. DOI: 10.1002/14651858.CD002281.pub3.

14. Klukowska M, Sharma N, Qaqish J, et al. Gingivitis reduction from a power brush with a novel brush head (Oral-B ${ }^{\circ}$ Precision CleanTM). J Dent Res. 2010;89:3695.

15. Klukowska M, Render JM, Timm H. A single-brushing study to compare plaque removal efficacy of a new power brush to an ADA reference manual toothbrush. Am J Dent. 2012;25(Special Issue A):1A-32A.

16. Kulkarni P, Kumar Singh D, Jalaluddin M. Comparison of efficacy of manual and powered toothbrushes in plaque control and gingival inflammation: A clinical study among the population of East Indian Region. J Int Soc Prev Community Dent. 2017;7(4):168-74.

17. Sharma NC, Qaqish J, Klukowska M, et al. The plaque removal efficacy of a novel power brush head. J Clin Dent. 2011;22(1):19-22.

18. Barnes CM, Weatherford TW 3rd, Menaker L. A comparison of the Braun Oral-B Plaque Remover (D5) electric and a manual toothbrush in affecting gingivitis. J Clin Dent. 1993;4(2):48-51.

19. Ganss C, Duran R, Winterfeld T, et al. Tooth brushing motion patterns with manual and powered toothbrushes -a randomised video observation study. Clin Oral Invest. 2017. DOI:10.1007/s00784-017-2146-7.

Corresponding author

prof dr hab. Jolanta Szymańska

Chair and Department of Paedodontics,

Medical University of Lublin,

7 Karmelicka St., 20-081 Lublin, Poland

E-mail: szymanska.lublin@gmail.com 